\title{
A VALUATION CRITERION FOR NORMAL BASES IN ELEMENTARY ABELIAN EXTENSIONS
}

\author{
NIGEL P. BYOTT AND G. GRIFFITH ELDER
}

\begin{abstract}
Let $p$ be a prime number and let $K$ be a finite extension of the field $\mathbb{Q}_{p}$ of $p$-adic numbers. Let $N$ be a fully ramified, elementary abelian extension of $K$. Under a mild hypothesis on the extension $N / K$, we show that every element of $N$ with valuation congruent $\bmod [N: K]$ to the largest lower ramification number of $N / K$ generates a normal basis for $N$ over $K$.
\end{abstract}

\section{INTRODUCTION}

The Normal Basis Theorem states that in a finite Galois extension $N / K$ there are elements $\alpha \in N$ whose conjugates $\{\sigma \alpha: \sigma \in \operatorname{Gal}(N / K)\}$ provide a vector space basis for $N$ over $K$. If $K$ is a finite extension of the field $\mathbb{Q}_{p}$ of $p$-adic numbers, the valuation $v_{N}(\alpha)$ of an element $\alpha$ of $N$ is an important property. We therefore ask whether anything can be said about the valuation of normal basis generators in this case. We will prove

Theorem 1. Let $K$ be a finite extension of the p-adic numbers, let $N / K$ be a fully ramified, elementary abelian $p$-extension, and let $b_{\max }$ denote the largest lower ramification number. If the upper ramification numbers of $N / K$ are relatively prime to $p$, then every element $\alpha \in N$ with valuation $v_{N}(\alpha) \equiv b_{\max } \bmod [N: K]$ generates a normal field basis. Moreover, no other equivalence class has this property: given any integer $v$ with $v \not \equiv b_{m} \bmod [N: K]$, there is an element $\rho_{v} \in N$ with $v_{N}\left(\rho_{v}\right)=v$ which does not generate a normal basis.

This result arose out of work on the Galois module structure of ideals in extensions of $p$-adic fields. For such extensions, it has been found that the usual ramification invariants are, in general, insufficient to determine Galois module structure, and thus that there is a need for a refined ramification filtration $\mathrm{BE} 02, \mathrm{BE05}, \mathrm{BE}$. This refined filtration is defined for elementary abelian $p$-extensions and requires elements that generate normal field bases. Such elements are provided by Theorem 1. Recent work Eld suggests that what is known for $p$-adic fields should also hold in the analogous situation in characteristic $p$, where $K$ is a finite extension of $\mathbb{F}_{p}(X)$. Here $\mathbb{F}_{p}$ denotes the finite field with $p$ elements, and $X$ is an indeterminate. We therefore make the

Conjecture. Theorem 1 holds when $K$ is a finite extension of $\mathbb{F}_{p}(X)$ as well.

Date: September 26, 2006.

1991 Mathematics Subject Classification. 11S15, $13 \mathrm{~B} 05$.

Key words and phrases. Normal Basis Theorem, Ramification Theory.

Elder was partially supported by NSF grant DMS-0201080. 


\section{Preliminary Results}

Let $K$ be a finite extension of the field $\mathbb{Q}_{p}$ of $p$-adic numbers, and let $N / K$ be a fully ramified, elementary abelian $p$-extension with $G=\operatorname{Gal}(N / K) \cong C_{p}^{n}$. Use subscripts to denote field of reference. So $\pi_{N}$ denotes a prime element in $N$, $v_{N}$ denotes the valuation normalized so that $v_{N}\left(\pi_{N}\right)=1$, and $e_{K}$ denotes the absolute ramification index. Let $\operatorname{Tr}_{N / K}$ denote the trace from $N$ down to $K$. For each integer $i \geq-1$, let $G_{i}=\left\{\sigma \in G: v_{N}\left((\sigma-1) \pi_{N}\right) \geq i+1\right\}$ be the $i$ th ramification group [Ser79, IV, §1]. Then $G_{-1}=G_{0}=G_{1}=G$, and the integers $b$ such that $G_{b} \supsetneq G_{b+1}$ are the lower ramification break (or jump) numbers. The collection of such numbers, $b_{1}<\cdots<b_{m}$, is the set of lower breaks. They satisfy $b_{1} \equiv \cdots \equiv b_{m} \bmod p\left[\operatorname{Ser79}\right.$, IV,$\S 2$, Prop. 11], where if $b_{m} \equiv 0 \bmod p$ then the extension $N / K$ is cyclic [Ser79, IV, $\S 2$, Ex. 3]. Let $g_{i}=\left|G_{i}\right|$. Then the upper ramification break numbers $u_{1}<\cdots<u_{m}$ are given by $u_{1}=b_{1} g_{b_{1}} / p^{n}=b_{1}$ and $u_{i}=\left(b_{1} g_{b_{1}}+\left(b_{2}-b_{1}\right) g_{b_{2}}+\cdots+\left(b_{i}-b_{i-1}\right) g_{b_{i}}\right) / p^{n}$ for $i \geq 2$ [Ser79, IV, §3].

Now by the Normal Basis Theorem, the set

$$
\mathcal{N B}=\left\{\rho \in N: \sum_{\sigma \in G} K \cdot \sigma \rho=N\right\}
$$

of normal basis generators is nonempty. We desire integers $v \in \mathbb{Z}$ such that $\{\rho \in$ $\left.N: v_{N}(\rho)=v\right\} \subset \mathcal{N B}$. And so we are concerned by the following

Example 1. Suppose $K$ contains a $p$ th root of unity $\zeta$, and let $N=K(x)$ with $x^{p}-\pi_{K}=0$. Let $\sigma$ generate $\operatorname{Gal}(N / K)$. Observe that $(\sigma-1) x^{p i}=0$ and $\operatorname{Tr}_{N / K} x^{i}=$ 0 for $p \nmid i$. So for each $i \in \mathbb{Z}$, we have $v_{N}\left(x^{i}\right)=i$ and $x^{i} \notin \mathcal{N B}$. Here $N / K$ has one ramification break $b=p e_{K} /(p-1)$, which is divisible by $p$. Ser79, IV, §2, Ex. 4].

Remark. Fortunately, these extensions provide the only obstacle. The restriction in Theorem 1 to elementary abelian extensions with upper ramification numbers relatively prime to $p$ is a restriction to those extensions that do not contain a cyclic subfield such as in Example 1 [Ser79, IV, §3 Prop. 14].

To prove Theorem 1 we need two results.

Lemma 2. Let $N / K$ be as above with $b_{m} \not \equiv 0 \bmod p$, and let $t_{G}=\sum_{i=1}^{m} b_{i}$. $\left|G_{b_{i}} \backslash G_{b_{i}+1}\right|$. If $\rho \in N$ with $v_{N}(\rho) \equiv b_{m} \bmod p^{n}$, then $v_{N}\left(\operatorname{Tr}_{N / K} \rho\right)=v_{N}(\rho)+t_{G}$. Conversely, given $\alpha \in K$ there is a $\rho \in N$ with $v_{N}(\rho)=v_{N}(\alpha)-t_{G} \equiv b_{m} \bmod p^{n}$ such that $\operatorname{Tr}_{N / K}(\rho)=\alpha$.

Proof. Use induction. Consider $n=1$ when $\operatorname{Gal}(N / K)=\langle\sigma\rangle$ is cyclic of degree $p$. There is only one break $b$, which satisfies $b<p e_{K} /(p-1)$. Given $\rho \in N$ with $v_{N}(\rho) \equiv b \bmod p$, we have $\operatorname{Tr}_{N / K} \rho \equiv(\sigma-1)^{p-1} \rho \bmod p \rho$. Since $(p-1) b<p e_{K}$, $v_{N}\left(\operatorname{Tr}_{N / K} \rho\right)=v_{N}(\rho)+(p-1) b$. And given $\alpha \in K$, use Ser79, V, §3, Lem. 4] to find $\rho \in N$ with $v_{N}(\rho)=v_{N}(\alpha)-(p-1) b$ and $\operatorname{Tr}_{N / K} \rho=\alpha$.

Assume now that the result is true for $n$, and consider $N / K$ to be a fully ramified abelian extension of degree $p^{n+1}$. Recall $g_{i}=\left|G_{i}\right|$. Let $H$ be a subgroup of $G$ of index $p$ with $G_{b_{2}} \subseteq H$. Let $L=N^{H}$ and note that $N / L$ satisfies our induction hypothesis. Moreover the ramification filtration of $H$ is given by $H_{i}=G_{i} \cap H$ Ser79, IV, §1]. So $\left|H_{i}\right|=g_{i}$ for $i>b_{1}$. Therefore $t_{H}=b_{m}\left(g_{b_{m}}-1\right)+b_{m-1}\left(g_{b_{m-1}-}\right.$ $\left.g_{b_{m}}\right)+\cdots+b_{1}\left(p^{n}-g_{b_{2}}\right)$. Given $\rho \in N$ with $v_{N}(\rho) \equiv b_{m} \bmod p^{n+1}$, by induction $v_{N}\left(\operatorname{Tr}_{N / L} \rho\right)=v_{N}(\rho)+t_{H}$. By the Hasse-Arf Theorem, $p^{n+1} \mid g_{b_{i}}\left(b_{i}-b_{i-1}\right)$ for $1 \leq$ 
$i \leq m$. Thus $t_{H} \equiv-b_{m}+p^{n} b_{1} \bmod p^{n+1}$ and $v_{L}\left(\operatorname{Tr}_{N / L} \rho\right) \equiv b_{1} \bmod p$. Using Ser79, IV, $\S 1$, Prop. 3 Cor.], $b_{1}$ is the Hilbert break for the $C_{p}$-extension $L / K$. Applying the case $n=1$, we find $v_{N}\left(\operatorname{Tr}_{N / K} \rho\right)=v_{N}(\rho)+t_{H}+p^{n}(p-1) b_{1}=v_{N}(\rho)+t_{G}$. The converse statement follows similarly, using $t_{H}+p^{n}(p-1) b_{1}=t_{G}$.

The following generalizes a technical relationship used in the proof of Lemma 2 .

Lemma 3. Let $N / K$ be a fully ramified, noncyclic, elementary abelian extension with group $G \cong C_{p}^{n}$. Let $H$ be a subgroup of $G$ of index $p$, and let $L=N^{H}$. If $b_{m}$ is the largest lower break of $N / K, b$ the only break of $N / L$, and $\rho$ any element of $N$ with $v_{N}(\rho) \equiv b_{m} \bmod p^{n}$, then $v_{L}\left(\operatorname{Tr}_{N / L} \rho\right) \equiv b \bmod p$.

Proof. In the proof of Lemma $2, H \supseteq G_{b_{2}}$ so that $G_{b_{1}} H / H \subsetneq G_{b_{1}+1} H / H$ following Ser79, IV, §1, Prop. 3, Cor.], and the break for $G / H$ was $b_{1}$. Here we have no such luxury and we have to involve the upper numbers in our considerations, although the argument is really no different. Note that there is a $k$ such that $G^{u_{k}+1} H / H \subsetneq G^{u_{k}} H / H$. Thus $u_{k}$ is the upper ramification number of $G / H$. Since there is only one break in the filtration of $G / H$, the lower and upper numbers for $G / H$ are the same, $b=u_{k}$.

The ramification filtration for $H$ is given by taking intersections: $H_{j}=G_{j} \cap H$. Note that $\left[G_{b_{i}}: G_{b_{i}} \cap H\right]=p$ for $i \leq k$ and $G_{b_{i}} \subseteq H$ for $i>k$. Let $h_{j}=\left|H_{j}\right|$. Then $h_{j}=g_{j} / p$ for $j \leq b_{k}$, and $h_{j}=g_{j}$ for $j>b_{k}$. Now let $v_{N}(\rho)=b_{m}+p^{n} t$. Following the proof of Lemma 2 and using the Hasse-Arf Theorem,

$$
\begin{gathered}
v_{N}\left(\operatorname{Tr}_{N / L} \rho\right)=b_{m}+p^{n} t+b_{m}\left(h_{b_{m}}-1\right)+b_{m-1}\left(h_{b_{m-1}}-h_{b_{m}}\right)+\cdots+b_{1}\left(h_{b_{1}}-h_{b_{2}}\right) \\
=p^{n} t+\left(b_{m}-b_{m-1}\right) h_{b_{m}}+\left(b_{m-1}-b_{m-2}\right) h_{b_{m-1}}+\cdots+\left(b_{2}-b_{1}\right) h_{b_{2}}+b_{1} h_{b_{1}} \\
\equiv\left(b_{k}-b_{k-1}\right) h_{b_{k}}+\cdots+\left(b_{2}-b_{1}\right) h_{b_{2}}+b_{1} h_{b_{1}} \equiv p^{n} u_{k} / p \equiv p^{n-1} b \bmod p^{n}
\end{gathered}
$$

Therefore $v_{L}\left(\operatorname{Tr}_{N / L} \rho\right) \equiv b \bmod p$.

\section{MAin Result}

Proof of Theorem 1. There are two statements to prove. We begin with the first: We assume the upper breaks satisfy $p \nmid u_{i}$, and prove that for $\rho \in N$

$$
v_{N}(\rho) \equiv b_{m} \bmod p^{n} \Longrightarrow \rho \in \mathcal{N} \mathcal{B} \text {. }
$$

The argument breaks up into two cases: the Kummer case where $\zeta \in K$ and the non-Kummer case where $\zeta \notin K$. Here $\zeta$ is a nontrivial $p$ th root of unity.

We begin with the Kummer case, and start with $n=1$. Let $\sigma$ generate the Galois group, and denote the one ramification number by $b$. Since in this case $b$ is also the upper number, $p \nmid b$. Therefore $\left\{v_{N}\left((\sigma-1)^{i} \rho: 0 \leq i<p\right\}\right.$ is a complete set of residues modulo $p$. And since $N / K$ is fully ramified, $\rho$ generates a normal basis. Now let $n \geq 2$ and note that $N=K\left(x_{1}, x_{2}, \ldots, x_{n}\right)$ with each $x_{i}^{p} \in K$. It suffices to show that $K[G] \rho$ contains each element $y=x_{1}^{j_{1}} x_{2}^{j_{2}} \cdots x_{n}^{j_{n}}$ with $0 \leq j_{i} \leq p-1$. For $y=1$ this is clear, since $\operatorname{Tr}_{N / K}(\rho) \in K$. For any other $y$, let $L=K(y)$ and let $b$ denote the ramification number of $L / K$. By Lemma $3, v_{L}\left(\operatorname{Tr}_{N / L}(\rho)\right) \equiv b \bmod p$. Since $b$ is an upper number of the ramification filtration of $G, p \nmid b$. Now apply the $n=1$ argument, using $\operatorname{Tr}_{N / L}(\rho)$ in $L / K$. Thus $y \in K[G] \rho$.

We now turn to the non-Kummer case with $\zeta \notin K$. Let $E=K(\zeta)$, let $E / K$ have ramification index $e_{E / K}$, and let $F=N(\zeta)$. Then $F / E$ is a fully ramified Kummer extension of degree $p^{n}$. Applying Herbrand's Theorem [Ser79, IV, §3, Lem. 5] to 
the quotient $G=\operatorname{Gal}(N / K)$ of $\operatorname{Gal}(F / K)$, we find that the maximal ramification break of $F / E$ is $e_{E / K} b_{m} \not \equiv 0 \bmod p$. The above discussion for the Kummer case therefore applies to $F / E$. Suppose now for a contradiction that $\rho \in N$ with $v_{N}(\rho) \equiv$ $b_{m} \bmod p^{n}$, and that $K[G] \rho$ is a proper subspace of $N$. Then by extending scalars (noticing that $E$ and $N$ are linearly disjoint as their degrees are coprime) we have that $E[G] \rho$ is a proper subspace of $F$. Moreover $v_{F}(\rho) \equiv e_{E / K} b_{m} \bmod p^{n}$. This contradicts the result already shown for the Kummer extension $F / K$, completing the proof of the first statement of the theorem.

Consider the second statement: Given any integer $v$ with $v \not \equiv b_{m} \bmod p^{n}$ there is a $\rho_{v} \in N$ with $v_{N}\left(\rho_{v}\right)=v$ such that $\operatorname{Tr}_{N / K} \rho_{v}=0$ and thus $\rho_{v} \notin \mathcal{N} \mathcal{B}$.

To prove this statement note that given $v \in \mathbb{Z}$, there is an $0 \leq a_{v}<p^{n}$ such that $v \equiv a_{v} b_{m} \bmod p^{n}$, since $p \nmid b_{m}$. If $a_{v} \neq 1$ we will construct an element $\rho_{v} \in N$ with $v_{N}\left(\rho_{v}\right)=v$ and $\operatorname{Tr}_{N / K} \rho_{v}=0$. To begin, observe that there is a integer $k$ such that $0 \leq k \leq n-1, a_{v} \equiv 1 \bmod p^{k}$ and $a_{v} \not \equiv 1 \bmod p^{k+1}$. Recall $g_{i}=\left|G_{i}\right|$. Since the ramification groups are $p$-groups with $g_{i+1} \leq g_{i}$, there is a Hilbert break $b_{s}$ such that $g_{b_{s}+1}<p^{k+1} \leq g_{b_{s}}$. For $i=k, k+1$ choose $H_{i}$ with $\left|H_{i}\right|=p^{i}$ and $G_{b_{s}+1} \subset$ $H_{k} \subset H_{k+1} \subseteq G_{b_{s}}$. Recall from Lemma 2 the expression for $t_{G}$, and note that $t_{H_{k}}=b_{m}\left(g_{b_{m}}-1\right)+b_{m-1}\left(g_{b_{m-1}}-g_{b_{m}}\right)+\cdots+b_{s}\left(p^{k}-g_{b_{s+1}}\right) \equiv-b_{m}+b_{s} p^{k} \bmod p^{n}$. Let $L=N^{H_{k}}$. Since $a_{v} \not \equiv 1 \bmod p^{k+1}, a_{v} \equiv 1+r p^{k} \bmod p^{k+1}$ for some $1 \leq r \leq p-1$. Using the fact that $b_{s} \equiv b_{m} \bmod p, a_{v} b_{m}+t_{H_{k}} \equiv(r+1) b_{m} p^{k} \bmod p^{k+1}$. Since $p^{k} \mid v_{N}(\alpha)$ for $\alpha \in L$, we can choose $\alpha \in L$ with $v_{N}(\alpha)=v+t_{H_{k}}-r p^{k} b_{s}$. So $v_{L}(\alpha) \equiv b_{s} \bmod p$. Let $\sigma \in G$ so that $\sigma H_{k}$ generates $H_{k+1} / H_{k}$. Therefore $v_{N}\left((\sigma-1)^{r} \alpha\right)=v+t_{H_{k}}$. Now using Lemma 2, we choose $\rho_{v} \in N$ such that $v_{N}\left(\rho_{v}\right)=v$ and $\operatorname{Tr}_{N / L} \rho_{v}=(\sigma-1)^{r} \alpha$. Since $\left(1+\sigma+\ldots+\sigma^{p-1}\right) \operatorname{Tr}_{N / L} \rho_{v}=0$, we have $\operatorname{Tr}_{N / K} \rho_{v}=0$.

Corollary 4. Let $N / K$ be a fully ramified, elementary abelian extension of degree $p^{n}$ with $n>1$ and one ramification break, at $b$. If $\rho \in N$ with $v_{N}(\rho) \equiv b \bmod p^{n}$, then $\rho \in \mathcal{N B}$.

\section{REFERENCES}

[BE] N. P. Byott and G. G. Elder, On the necessity of new ramification breaks, In preparation.

[BE02] N. P. Byott and G. G. Elder, Biquadratic extensions with one break, Can. Math. Bull. 45 (2002), no. 2, 168-179.

[BE05] N. P. Byott and G. G. Elder, New ramification breaks and additive Galois structure, J. Théor. Nombres Bordeaux 17 (2005), no. 1, 87-107.

[Eld] G. G. Elder, One-dimensional elementary-abelian extensions of local fields, arXiv.org:math/0511174 (2005-11-15).

[Ser79] J.-P. Serre, Local fields, Springer-Verlag, New York, 1979.

Nigel P. Byott, School of Engineering, Computer Science and Mathematics, UniverSity of Exeter, EXeter EX4 4QE, United Kingdom

E-mail address: N.P.Byott@ex.ac.uk

G. Griffith Elder, Department of Mathematics, University of Nebraska at Omaha, OMAHA, NE 68182-0243 U.S.A.

E-mail address: elder@vt.edu

Current address: Department of Mathematics, Virginia Tech, Blacksburg VA 24061-0123 